\title{
Design and Development of Three Arms Transmission Line Inspection Robot
}

\author{
Muhammad Fairuz Abdul Jalal*, Khairul Salleh Mohamed Sahari, Ho Ming Fei, Justin Chan Tuck Leong \\ Department of Mechanical Engineering, College of Engineering, Universiti Tenaga Nasional, Kajang, Selangor 43000, Malaysia
}

\section{ARTICLE INFO}

Article History

Received 19 July 2018

Accepted 12 November 2018

\section{Keywords}

Inspection robot

transmission line

slider

\begin{abstract}
The high-voltage transmission line had been used primarily for power distribution from power plant or power station to the end users. However, the transmission line is highly prone to damage due to exposure to various thermal-mechanical loadings and material degradation. Therefore, periodical inspection on transmission line after prolonged service is needed to prevent any failure before it happens. In this paper, we present a new design of three arms inspection robot for transmission lines. The robot is able to transverse along the line and bypass the in-line obstacles namely the anti-vibration hammers, spacer, strain clamps and others. The design of the inspection robot in term of the robot design and configuration with slotted cam at each arm is presented. The detailed analysis via simulation with respect to the robot stability; kinematic and movement analysis; and power consumption during operation is executed to make sure the proposed design able to do the inspection without any unexpected difficulties. Later, the lab testing on the developed prototype is done for feasibility study and validation.
\end{abstract}

(c) 2018 The Authors. Published by Atlantis Press SARL. This is an open access article under the CC BY-NC license (http://creativecommons.org/licenses/by-nc/4.0/).

\section{INTRODUCTION}

High-voltage transmission lines is one of the main elements in power distribution from the power plant or power station to the customer. However, the transmissions line is exposed to various environment conditions namely thermo-mechanical loading, mechanical tension, material degradation and material corrosion. The transmissions lines undergoing such circumstances eventually lead to many problems such as electrical breakdown or even major accident if transmissions lines were not being inspect, fixed and replaced in appropriate time [1].

The process of inspections of transmission line involved activities such as changing the ceramic insulators and switching on or off the circuit in between poles [2]. The manual inspection routine of transmission line is very tedious, dangerous and time-consuming inspection session [3]. The development of the mobile inspection robot will not only create a safer work environment in the transmission line inspection job, but also improve the efficiency of the inspection process [3].

Various line inspection robots have been developed and proposed by researchers. Wang et al. [4] proposed a two arms transmission line robot. This robot utilized a line walking mechanism that based on biped structure either having both feet placed on the line or each feet is placed alternatively on and off the line. Another dual arm robot self-balancing was developed by Songyi et al. [5]. The two arm of the robot hold onto the transmission line and move with the wheel installed on the arm. The robot is integrated with counter

*Corresponding author.Email: MFairuz@uniten.edu.my weight to improve the robot stability while travelling on the transmission line.

$\mathrm{Xu}$ et al. [6] developed a three arms transmission line robot that features two supporting arms and one assistant arm placed in between both supporting arms. This robot was designed to overcome obstacles by evading them with the lifting mechanism on each arm. Rui et al. [7] developed three arm transmission line inspection robot. The robot arms consist of rubber wheel and the both outer arm installed with gripper to hold unto the line. The rubber wheel enables the robot to bypass minor obstacles for instance the splicer and damper.

Our research group already took initiatives to propose various conceptual design and selection based on requirement set by Tenaga Nasional Berhad, the main utility supplier to peninsular Malaysia [8]. In this paper, the prototype of a three arms transmission line inspection robot is presented. The robot has the capabilities to pass over in-line obstacles without deteriorating the robot stability.

\section{TRANSMISSION LINE INSPECTION ROBOT DESIGN}

\subsection{General Design Requirement}

There are many components, which are installed on the transmission line for various purposes namely tension insulator chain, suspension clamp, damper, tension clamp, jumper, splicing sleeve and others depending on type of transmission line as shown in Figure 1. 
It is deemed necessary for robot to bypass these in-line components so that the inspection process can be executed effectively [9].

\subsection{Transmission Line Inspection Robot}

The robot travel on the transmission line with the help of rollers that installed in each arm and cross obstacles by moving its arm up by rotating the power screw installed in each arm. The robot is installed with six motors; three motors run the roller at each arm for traversing on the transmission line. The others three motors are attached to the power screw for bypassing the in-line obstacles. The conceptual design and the prototype are shown in Figure 2.

The three arms robot is hanged to the transmission line by means of roller and lower jaw as a griping element. The movement is propelled by the roller at each arm. Each roller is powered by a motor as shown in Figure 3.

The mechanism to bypass obstacle is illustrated in Figure 4. The gripping position and bypassing obstacle movement are realized by the rotational direction of the motor linked to power screw. To bypass the in-line obstacle, the motor is then actuated. The power screw pushes the arm upwards. As the arm moves up, it follows

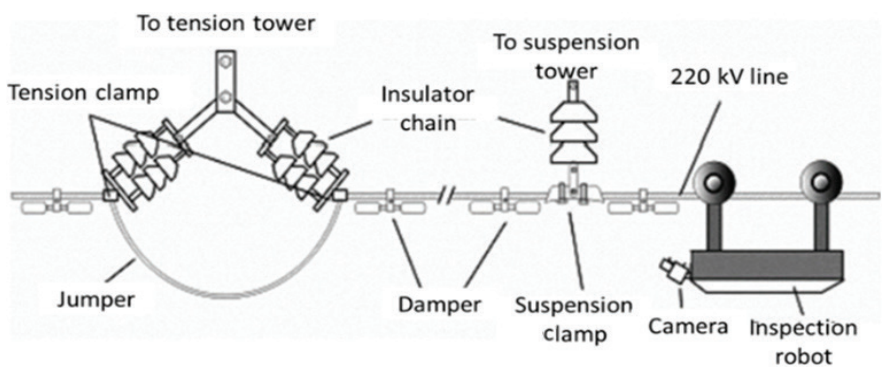

Figure 1 Transmission line environment [9]
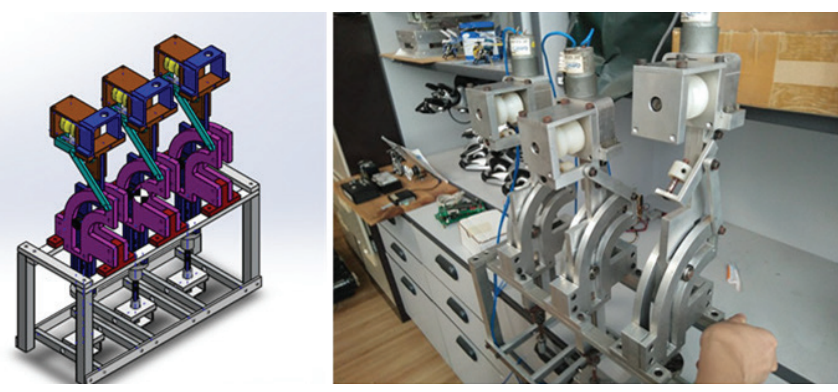

Figure 2 The conceptual design (left) and prototype of the three arms transmission line inspection robot (right)
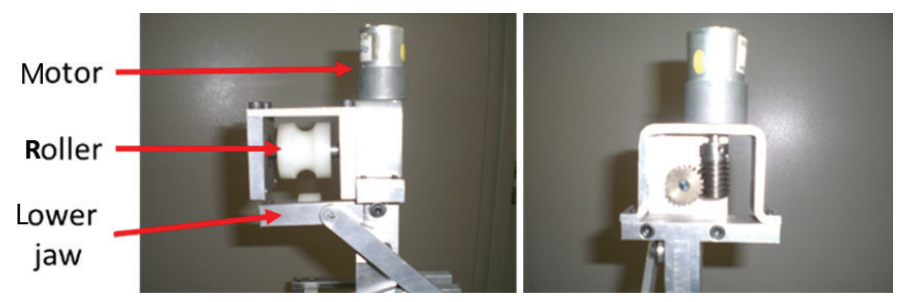

Figure 3 The front view (left) and side view (right) of the gripper the curve of the slotted profile. The bottom of the arm will have a pin joint with a support to enable it to rotate as the arm follows the curve of the slot. The arm will move back to its original position by reversing the motor direction and the wheel on arm attached back to the line. The same step applied to the following next two arm. The mechanism to bypass the obstacles is shown in Figure 5.

The stability of the transmission line inspection robot is an important aspect to be considered as it influences the overall quality of the image taken during the inspection process.

\section{RESULT AND DISCUSSION}

\subsection{Capability to Bypass Obstacles}

The robot must be able to avoid obstacles along the transmission line or else the design will be deemed as a failure. Figure 6 shows that the roller and lower jaw do not interfere with the line as the arm gripper loosening and tightening its grip to the transmission line.

The clearance between the arm and the obstacles is ranged from 90 to $130 \mathrm{~mm}$ and the maximum angle of arm rotation $21.6^{\circ}$ as shown in Figure 7.

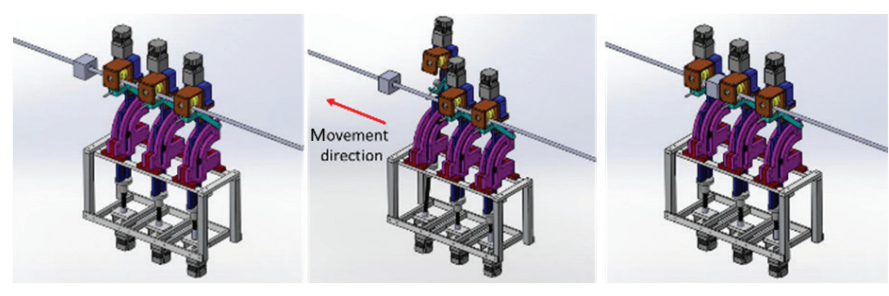

Figure 4 Mechanism to bypass in-line obstacles
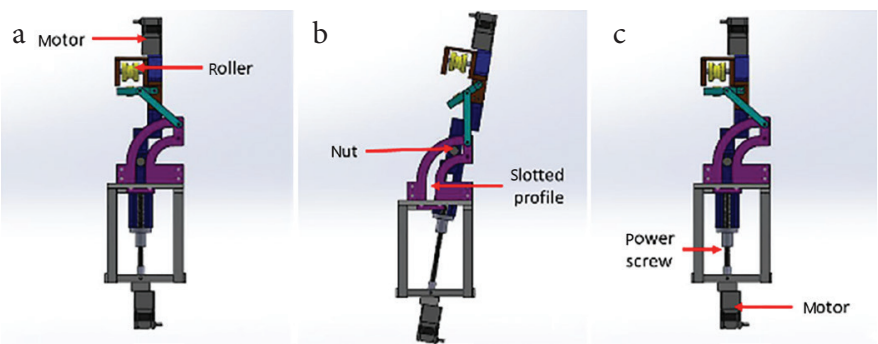

Figure 5 The arm movement to bypass obstacles. (a) initial state (b) obstacle bypassing state (c) return back to initial state
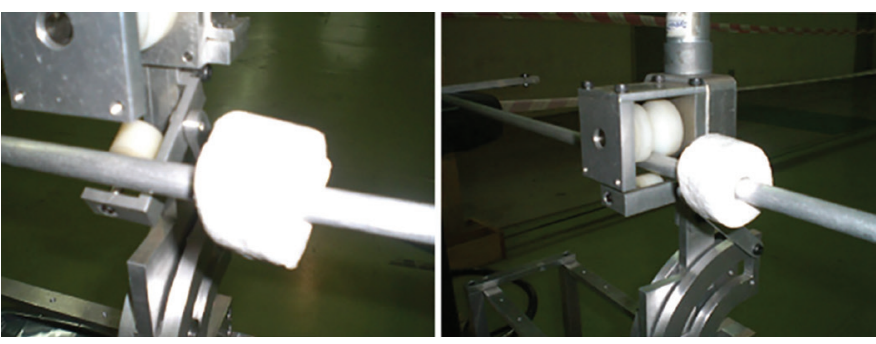

Figure 6 The arm gripper during loosening (left) and tightening (right) its grip to the line 

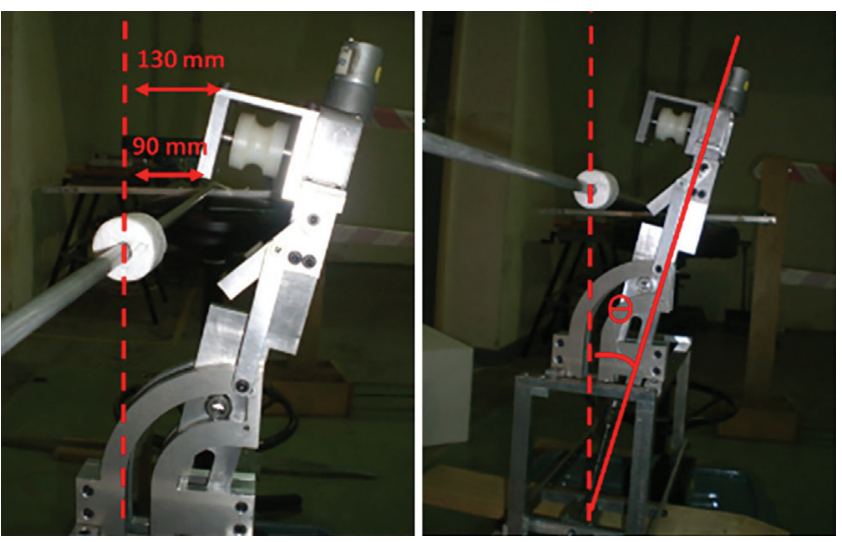

Figure $7 \mid$ The arm gripper during loosening (left) and tightening (right) its grip to the line

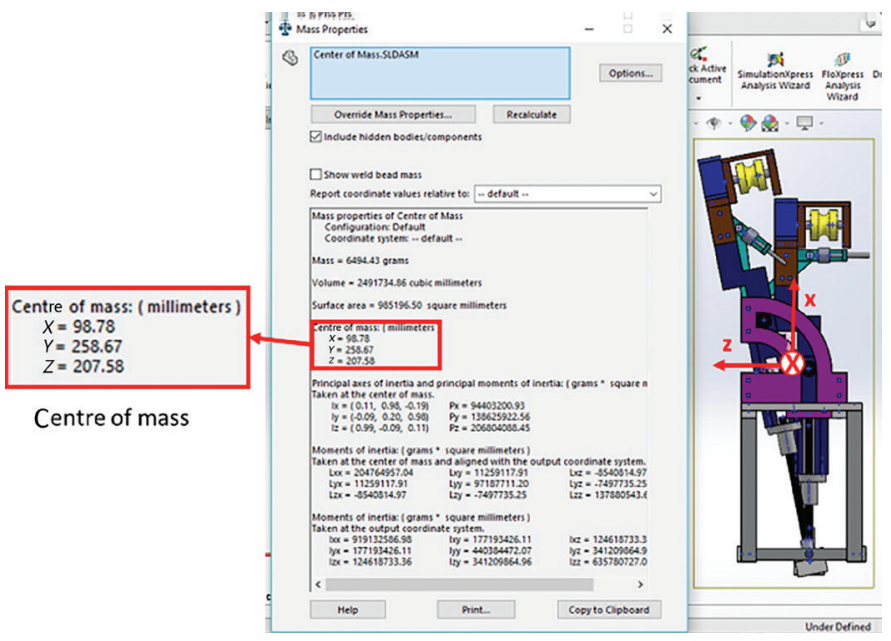

Figure 8 The coordinate center of mass extracted from simulation

\subsection{Stability Analysis}

The significant changes of robot center of mass for bypassing the obstacle provide instability and tendency for the robot to swing. The analysis of the design center of gravity is done by means of center of mass analysis of the following arm configurations:

(i) Configuration 1: Original configuration; all the arms rest on the transmission line.

(ii) Configuration 2: The first arm move up to avoid obstacles while the other two arm rest on the line.

(iii) Configuration 3: The second arm move up to avoid obstacles while the other two arm rest on the line.

(iv) Configuration 4: The third arm move up to avoid obstacles while the other two arm rest on the line.

The robot $X Y Z$ axis and the value of center of mass for each configuration is extracted from the CAD software is as shown in Figure 8. The coordinate center of mass of the different arm configuration is tabulated in Table 1.

There is a small change in the coordinate of the center of mass in the $X$ - and $Y$-axis as the robot change its configurations from the original configuration while the $Z$-axis remained constant throughout the operation. The average change of $X$-axis is $10.33 \mathrm{~mm}$ and $Y$-axis is $8.07 \mathrm{~mm}$. The average distance between center of mass in various arm configuration is $13.1 \mathrm{~mm}$. The results are plotted in $X-Y$ plane as shown in Figure 9.

\subsection{Motor Torque and Power Consumption}

The value of the motor torque required to rotate the power screw to move the arm up for bypassing the obstacle is analyzed in Solidworks Motion. The motor speed is set to reach $225 \mathrm{rpm}$ based on the motor specification. The time needed for the arm to move up to bypass the obstacle is $20 \mathrm{~s}$. The average motor torque and power consumption are $13 \mathrm{Nm}$ and $0.254 \mathrm{~W}$. The graph of the motor torque and power consumption against time is shown in Figure 10.

Table 1 The coordinate of the center of mass by various arm configuration

\begin{tabular}{lrrrr}
\hline \multirow{2}{*}{ Coordinate } & \multicolumn{5}{c}{ Arm configuration } \\
\cline { 2 - 5 } & \multicolumn{1}{c}{$\mathbf{1}$} & \multicolumn{1}{c}{$\mathbf{2}$} & \multicolumn{1}{c}{$\mathbf{3}$} & \multicolumn{1}{|c}{$\mathbf{4}$} \\
\hline$X$ & 88.45 & 98.78 & 98.80 & 98.80 \\
$Y$ & 250.65 & 258.67 & 258.83 & 258.83 \\
$Z$ & 207.58 & 207.58 & 207.58 & 207.58 \\
\hline
\end{tabular}

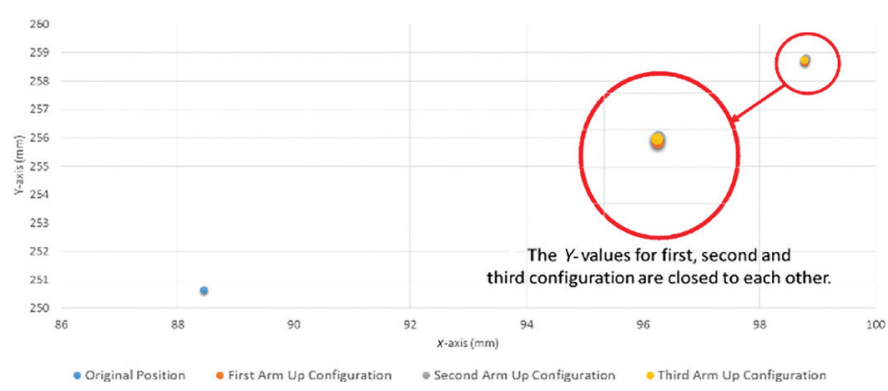

Figure 9 The coordinate of robot center of mass in $X-Y$ plane for different arm configuration
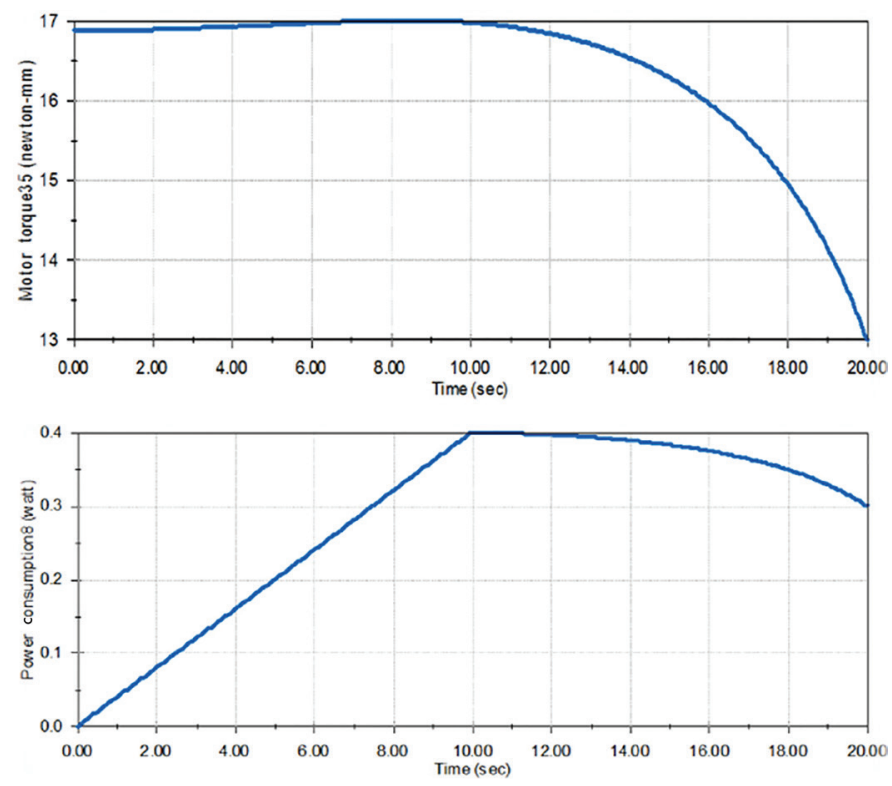

Figure $10 \mid$ Graph of motor torque (top) and power consumption (bottom) against time 


\section{CONCLUSION}

In this paper, the three arms transmission line inspection robot and the analysis of robot stability and motor are presented. The mechanism to bypass the in-line obstacles is achieved by using power screw and slotted profile. The robot is proven to be stabile during operation due to minimal changes of its centre of mass.

\section{ACKNOWLEDGMENTS}

This work was supported by the Universiti Tenaga Nasional Bold (TNB) Grant 102289176/B/9/2017/63 and Internal Grant J510050707. The author would like to thank the TNB for the access of information and data for formulating the robot design specification.

\section{REFERENCES}

[1] Y.C. Zhang, Z.Z. Liang, M. Tan, Mobile robot for overhead powerline inspection: a review, Robot 9 (2009), 467-473.

[2] L.E. Parker, J.V. Draper, Robotics applications in maintenance and repair, S. Nof, Handbook of Industrial Robotics, second ed., Hoboken, NJ, John Wiley \& Sons Inc., 1998, pp. 1023-1036.

[3] F.Y. Zhou, J.D. Wang, Y.B. Li, H.R. Xiao, Control of an inspection robot for $110 \mathrm{KV}$ power transmission lines based on expert system design methods, Proceedings of the 2005 IEEE

\section{Authors Introduction}

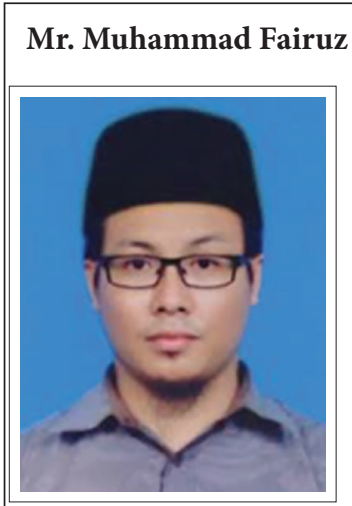

$\mathrm{He}$ is a lecturer at Department of Mechanical Engineering, Universiti Tenaga Malaysia. He received his Diplom -Ing. (FH) in Mechanical Engineering and MSc. in Mechatronics from University of Applied Sciences Ravensburg - Weingarten Germany in 2008 and 2010 accordingly.

His research interest include robotic, design methodology and inspection system for power industry. He is a member of International TRIZ Association.

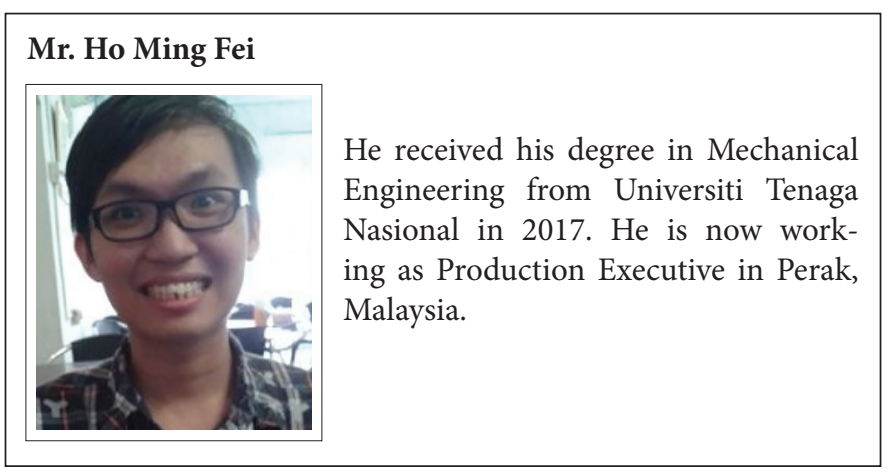

International Conference on Control Applications, IEEE, Toronto, ON, Canada, 2005, pp. 1563-1568.

[4] L. Wang, F. Liu, Z. Wang, S. Xu, S. Cheng, J. Zhang, Development of a practical power transmission line inspection robot based on a novel line walking mechanism, IEEE/RSJ International Conference on Intelligent Robots and Systems, IEEE, Taipei, Taiwan, 2010, pp. 222-227.

[5] D. Songyi, W. Xuefeng, D. Hang, W. Tao, Development of a selfbalance dual-arm robot for inspection of high-voltage power transmission lines, 2012 IEEE International Conference on Mechatronics and Automation, IEEE, Chengdu, China, 2012, pp. $2482-$ 2487.

[6] B. Xu, X. Wang, Y. Zhu, H. Chen, Design of obstacle crossing mechanism of high-voltage transmission line inspection robot, 2015 IEEE International Conference on Robotics and Biomimetics (ROBIO), IEEE, Zhuhai, China, 2015, pp. 2539-2544.

[7] G. Rui, Z. Feng, C. Lei, Y. Jun, A mobile robot for inspection of overhead transmission lines, Proceedings of the 2014 3rd International Conference on Applied Robotics for the Power Industry, IEEE, Foz do Iguassu, Brazil, 2014, pp. 1-3.

[8] M.F. Abdul Jalal, K.S. Mohamed Sahari, A. Anuar, A.D. Mohd Arshad, M.S. Idris, Conceptual design for transmission line inspection robot, IOP Conference Series: Earth and Environmental Science, Bristol, IOP Publishing Ltd, 2013, pp. 1-4.

[9] G. Wu, T. Zheng, H. Xiao, C. Li, Navigation, location and noncollision obstacles overcoming for high-voltage power transmissionline inspection robot, 2009 International Conference on Mechatronics and Automation, IEEE, Changchun, China, 2009, 2014-2020.
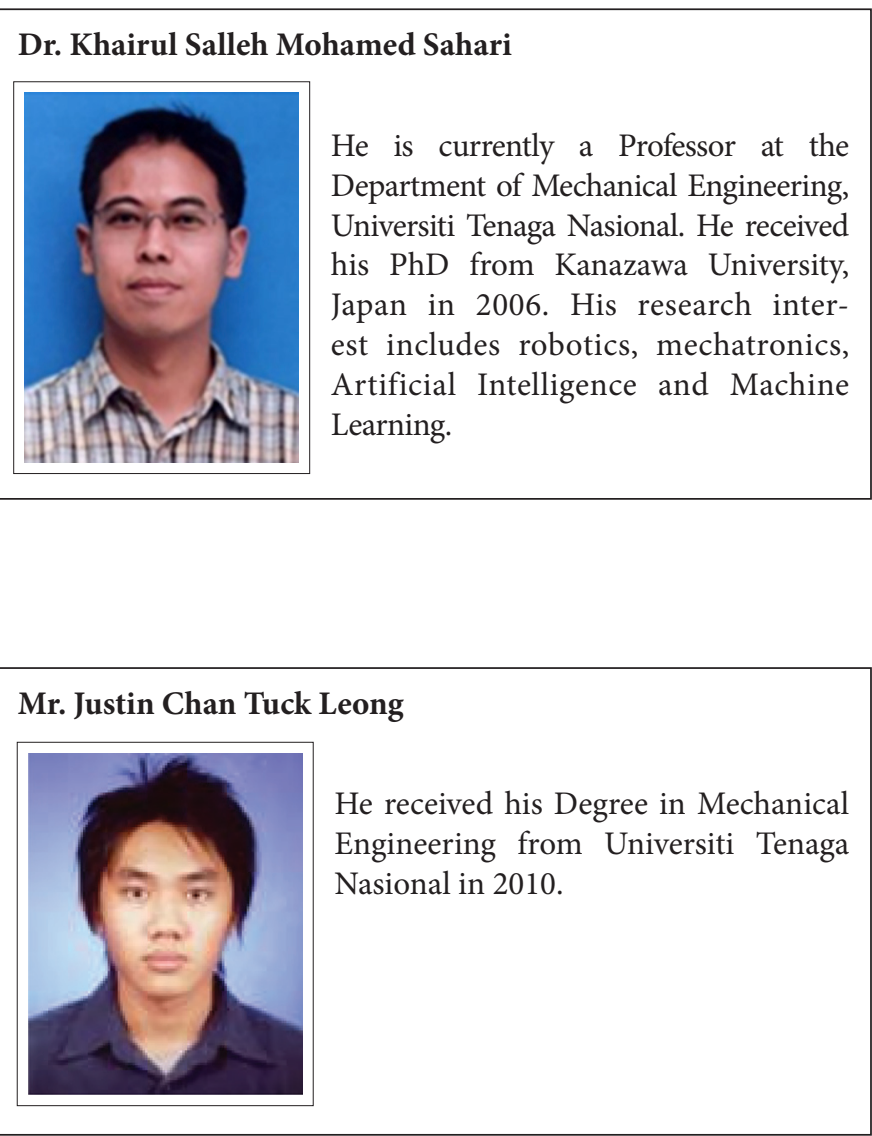